\title{
Cerebral deficiency of vitamin B5 (D-pantothenic acid; pantothenate) as a potentially-reversible cause of neurodegeneration and dementia in sporadic Alzheimer's disease \\ DOI:
}

10.1016/j.bbrc.2020.05.015

\section{Document Version}

Accepted author manuscript

Link to publication record in Manchester Research Explorer

\section{Citation for published version (APA):}

Xu, J., Patassini, S., Begley, P., Church, S., Waldvogel, H. J., Faull, R. L. M., Unwin, R. D., \& Cooper, G. J. S. (2020). Cerebral deficiency of vitamin B5 (D-pantothenic acid; pantothenate) as a potentially-reversible cause of neurodegeneration and dementia in sporadic Alzheimer's disease. Biochemical and Biophysical Research Communications, 527(3), 676-681. https://doi.org/10.1016/j.bbrc.2020.05.015

\section{Published in:}

Biochemical and Biophysical Research Communications

\section{Citing this paper}

Please note that where the full-text provided on Manchester Research Explorer is the Author Accepted Manuscript or Proof version this may differ from the final Published version. If citing, it is advised that you check and use the publisher's definitive version.

\section{General rights}

Copyright and moral rights for the publications made accessible in the Research Explorer are retained by the authors and/or other copyright owners and it is a condition of accessing publications that users recognise and abide by the legal requirements associated with these rights.

\section{Takedown policy}

If you believe that this document breaches copyright please refer to the University of Manchester's Takedown Procedures [http://man.ac.uk/04Y6Bo] or contact uml.scholarlycommunications@manchester.ac.uk providing relevant details, so we can investigate your claim.

\section{OPEN ACCESS}


FINAL VERSION [4584 words]

2 Cerebral deficiency of vitamin B5 (D-pantothenic acid; pantothenate) as a potentially-

3 reversible cause of neurodegeneration and dementia in sporadic Alzheimer's disease

4 Jingshu Xu ${ }^{1,2}$, Stefano Patassini ${ }^{1,2}$, Paul Begley ${ }^{1}$, Stephanie Church ${ }^{1}$, Henry J. Waldvogel ${ }^{3}$,

5 Richard L. M. Faull ${ }^{3}$, Richard D. Unwin ${ }^{1}$, and Garth J. S. Cooper ${ }^{1,2,3, *}$

6

$7 \quad{ }^{1}$ Division of Cardiovascular Sciences, School of Medical Sciences, Faculty of Biology,

8 Medicine and Health, The University of Manchester, Manchester, M13 9WL and Centre for

9 Advanced Discovery and Experimental Therapeutics (CADET), Central Manchester University

10 Hospitals NHS Foundation Trust, Manchester Academic Health Sciences Centre, Manchester,

11 UK

$12{ }^{2}$ School of Biological Sciences, Faculty of Science, University of Auckland, New Zealand.

$13{ }^{3}$ Centre for Brain Research, Faculty of Medical and Health Sciences, University of Auckland, 14 Auckland, New Zealand.

15

16 *Corresponding Author; e-mail garth.cooper@manchester.ac.uk.

17 Address for correspondence: Garth J S Cooper, Centre for Advanced Discovery and

18 Experimental Therapeutics, Division of Cardiovascular Sciences, School of Medical Sciences,

19 Faculty of Biology, Medicine \& Health, The University of Manchester M13 9NT

20 


\section{Abstract}

22 Alzheimer's disease (AD) is the most common cause of age-related neurodegeneration and

23 dementia, and there are no available treatments with proven disease-modifying actions. It is

24 therefore appropriate to study hitherto-unknown aspects of brain structure/function in AD

25 to seek alternative disease-related mechanisms that might be targeted by new therapeutic

26 interventions with disease-modifying actions. During hypothesis-generating metabolomic

27 studies of brain, we identified apparent differences in levels of vitamin B5 between AD cases

28 and controls. We therefore developed a method based on gas chromatography-mass

29 spectrometry by which we quantitated vitamin B5 concentrations in seven brain regions

30 from nine $A D$ cases and nine controls. We found that widespread, severe cerebral deficiency

31 of vitamin $B 5$ occurs in AD. This deficiency was worse in those regions known to undergo

32 severe damage, including the hippocampus, entorhinal cortex, and middle temporal gyrus.

33 Vitamin B5 is the obligate precursor of CoA/acetyl-CoA (acetyl-coenzyme A), which plays

34 myriad key roles in the metabolism of all organs, including the brain. In brain, acetyl-CoA is

35 the obligate precursor of the neurotransmitter acetylcholine, and the complex fatty-acyl

36 groups that mediate the essential insulator role of myelin, both processes being defective in

$37 \mathrm{AD}$; moreover, the large cerebral vitamin $\mathrm{B} 5$ concentrations co-localize almost entirely to

38 white matter. Vitamin B5 is well tolerated when administered orally to humans and other

39 mammals. We conclude that cerebral vitamin B5 deficiency may well cause

40 neurodegeneration and dementia in $A D$, which might be preventable or even reversible in its

41 early stages, by treatment with suitable oral doses of vitamin B5. 
43 Keywords: Alzheimer's disease; Human brain; Vitamin B5 (pantothenic acid; pantothenate);

44 Age-related neurodegeneration; Acetyl-CoA; Metabolic brain disease

45 
46 Abbreviations: AD, Alzheimer's disease; CoA, coenzyme A; GC-MS, gas chromatography-

47 mass spectrometry; PANK, pantothenate kinase; PDH, pyruvate dehydrogenase; PKAN,

48 pantothenate kinase-associated neurodegeneration; TCA, tricarboxylic acid

49 
51 Alzheimer's disease (AD) is characterised by neuropathological hallmarks of senile plaques

52 and neurofibrillary tangles in the brain, and clinically-insidious onset with progressive

53 decline of neurocognitive function. $A D$ is the most common cause of age-related

54 neurodegeneration and dementia and represents the largest unmet medical need in

55 neurology $[1,2]$.

56 The pathogenesis of $A D$ is complex, and metabolic abnormalities in the brain lie at the core

57 of its molecular pathology [3]. Well-documented metabolic disturbances associated with AD-

58 evoked neurodegeneration include: abnormal $A \beta$ and $\tau$ metabolism with the formation of

59 protein aggregates implicated in tissue damage; mitochondrial dysfunction; oxidative stress;

60 and impaired cerebral glucose uptake and energy metabolism. More recently, marked

61 elevations in glucose levels of AD brain have been reported by three independent studies

62 that employed different methodologies [4-6], and the evident global perturbation of

63 metabolites supports the notion of $A D$ as a pervasive metabolic disorder [7].

64 Our group's prior investigations have indicated that enzymes and metabolites involved in 65 glycolysis, the pentose-phosphate pathway, the polyol pathway, the tricarboxylic acid (TCA) 66 cycle, and oxidative phosphorylation are consistently affected in the AD brain when

67 compared with controls [7, 8]. Acetyl-CoA plays a central role in metabolism, functioning as 68 the necessary molecule through which glycolysis-derived pyruvate enters the tricarboxylic 69 acid (TCA) cycle through pyruvate dehydrogenase (PDH)-mediated catalysis [9]. CoA itself 70 not only forms the major integral aspect of the acetyl-CoA molecule but is also a cofactor for 71 numerous pathways such as oxidative respiration and lipid metabolism, and plays a 72 regulatory role in brain health through its involvement in the synthesis of neurotransmitters 73 such as acetylcholine [10], and in cerebral myelin biosynthesis [11]. For the biosynthesis of $74 \mathrm{CoA}$, vitamin $\mathrm{B} 5$ is essential as both a primary substrate and a rate-limiting metabolite, 75 whose utilization is catalysed by the enzyme pantothenate kinase (PANK) [17]. Mutations in 76 the gene PANK2, which encodes mitochondria-localized PANK2 (one of four human pantothenate kinases encoded in the human genome $[12,13])$, lead to PANK-associated neurodegeneration (PKAN), which strongly suggests the critical importance of vitamin B5 for maintaining neural health and integrity; this view is further supported by its known obligate roles in the biosynthesis of acetylcholine and of myelin [11].

81 Here we applied quantitative gas chromatography-mass spectrometry (GC-MS) to determine 82 putative alterations in vitamin $B 5$ concentrations in brain tissue from $A D$ cases and matched 
83 controls. We analysed 18 human brains with short post-mortem delay wherein seven

84 anatomically- and functionally-distinct brain regions were examined in each: three of these

85 are known to undergo severe neuronal damage in AD (hippocampus, entorhinal cortex, and

86 middle temporal gyrus); three to be moderately affected (cingulate gyrus, sensory cortex

87 and motor cortex); and one (cerebellum) thought to be relatively spared [14-16].

88 
2.1. Acquisition of human brains

91 All experiments were performed in accordance with relevant guidelines and regulations. This

92 case-control study of post-mortem brain was approved by the University of Auckland Human

93 Participants Ethics Committee with informed consent from all families.

94 Human brains were obtained from the Neurological Foundation Human Brain Bank,

95 University of Auckland, as detailed in the Suppl. Methods 2.1.

96

97

98

99

100

101

102

103

104

105

\subsection{Dissection of human brains}

Human brains were dissected as described in the Suppl. Methods 2.2.

\subsection{Metabolite extraction for GC-MS}

Metabolites were extracted for GC-MS as detailed in the Suppl. Methods 2.3.

\subsection{Sample preparation}

Samples were prepared for GC-MS as detailed in the Suppl. Methods 2.4.

\subsection{GC-MS}

GC-MS was performed as detailed in the Suppl. Methods 2.5 .

\subsection{Data analysis}

GC-MS data were analysed as described in the Suppl. Methods 2.6.

\subsection{Cerebral vitamin B5 concentrations measured by GC-MS}

The reproducibility and accuracy of our targeted vitamin B5 assay have been reported elsewhere [17]. Briefly, to determine vitamin B5 concentration by GC-MS, we generated a calibration curve using known concentrations of authentic synthetic standards (SigmaAldrich) in human-brain extract as matrix. We calculated vitamin B5 levels in individual brain samples using the calibration curve, and data have been presented here as $\mu \mathrm{mol} / \mathrm{kg}$ of fresh (wet) brain tissue. Calculation of metabolite abundance was performed on internal-standard ratios rather than raw peak areas in order to improve reproducibility.

\subsection{Statistical analysis}

Data were analysed using an in-house bioinformatic work-flow developed in R v3.5.1.

Statistical analysis and data visualization were performed using in-house scripts implemented in the following R packages: tidyr v0.8.1; ggplot2 v3.0.0; ROCR v1.0-7; nnet v7.3-12; MASS v7.3-50; lattice v0.20-35; and magrittr v1.5. A receiver-operating 
119 characteristic (ROC) curve was generated by combining all measurements in this study and

120 applying ROCR v1.0-7; nnet v7.3-12 was used to fit multinomial log-linear models via neural

121 networks.

122 To ensure the reproducibility of our assay, we first assessed the QC replicate samples and

123 evaluated the \% coefficient-of-variation (\%CV) of vitamin B5 measurements in each

124 experimental batch. We set the \%CV for QC samples of $15 \%$ as the cut-off for acceptable

125 reproducibility.

126 For each brain region, effects of age, post-mortem delay (PMD), and brain-weight on the

127 vitamin B5 concentrations in human brain were investigated by Spearman's correlation

128 analysis. A correlation was considered to be significant only if the correlation coefficient was

$129>0.8$ or $<-0.8$, and the corresponding P-value was $<0.01$.

130 
132 We measured vitamin B5 concentrations in each of 7 brain regions from 9 patients

133 with $\mathrm{AD}$ and 9 controls, with matching of cases and controls for age, sex, and post-

134 mortem delay (Table 1). Average brain weight of the AD group was significantly

135 lower than that of the control group; the observed decline in brain weight is in line

136 with the severity of neurodegeneration. All AD cases studied had Braak stages of IV

137 or above; one control had Braak stage II, which was retained within the control group

138 for analysis (Suppl. Table 1) as this finding is consistent with the known frequency of

139 asymptomatic AD in similarly-aged groups in the study population [18].

\section{Table 1}

Case-control study of post-mortem human brain in AD: group characteristics

\begin{tabular}{lcc}
\hline Variable & Control & Alzheimer's disease \\
\hline Number & 9 & 9 \\
Age & $70.1(6.7)$ & $70.3(7.1)$ \\
Male sex, & $5(55.6)$ & $5(55.6)$ \\
$\mathrm{n}(\%)$ & & 7 \\
PMD (h) & 9 & $(4.0-12.0)$ \\
Brain-weight & 1260 & 1062 \\
(g) & $(1094-1461)$ & $(831-1355)^{*}$ \\
Plaques, & $1(11)$ & $9(100)^{* *}$ \\
$\mathrm{n}(\%)$ & $1(11)$ & $9(100)^{* *}$ \\
Tangles, & \\
$\mathrm{n}(\%)$ &
\end{tabular}

Values are: age, mean (SD); PMD and brain-weights, median (range):

$* \mathrm{P}=0.005,{ }^{*} * \mathrm{P}<0.0001$ compared with corresponding Control value; all other differences are non-significant. Abbreviation: PMD, post-mortem delay 
141 To quantify vitamin B5 levels in human brain tissue, we generated a calibration curve

142 by constituting different concentrations of synthetic vitamin B5 as pure standard

143 solutions (Fig.1a) and also in human brain-derived background matrices (Fig.1b).

144 Vitamin B5 showed stable linearity $\left(\mathrm{R}^{2}>0.99\right)$ within the range at which we

145 measured brain concentrations; the standard curve shown in Fig. 1b was used to

146 interpolate the regional concentrations of vitamin B5 reported here.

147 We observed a global reduction in vitamin B5 levels across all 7 brain regions in 148 cases compared with controls (Fig. 2a). Control brains showed greater variation in 149 vitamin B5 levels in general, and regions corresponding to those heavily affected in 150 AD (hippocampus, entorhinal cortex, and middle temporal gyrus) showed lower mean 151 vitamin B5 values when compared to regions generally considered to be less severely 152 affected (sensory cortex, motor cortex, and cerebellum). The QC-replicate samples (N $153=8$ per region per condition) measured in this study showed low $\% \mathrm{CV}$ values across 154 all brain regions $(\max =12 \%)$ (Fig. $2 b)$, demonstrating the high degree of 155 reproducibility of our assay.

156 The decrease in cerebral vitamin B5 levels in AD cases was observed in all brain 157 regions studied, which are representative of the majority of brain mass (Fig. 2). The 158 range of mean vitamin B5 concentrations in control brains was between 33.7 (in 159 cingulate gyrus) and 55.4 (cerebellum) $\mu \mathrm{mol} / \mathrm{kg}$ of fresh brain tissue, and between 16015.2 (entorhinal cortex) and 25.4 (cerebellum) in AD. These data reflect copious 161 amounts of vitamin B5 within the normal brain. Fold-changes in vitamin B5 were 162 similar across all brain regions examined, showing an average of $60 \%$ reduction with 163 statistical significance typically being $\mathrm{P}<0.01$ (Fig. 2c).

164 Thus similar levels of vitamin B5 depletion are pervasive in the AD brain. This 165 finding is potentially consistent with comparable degrees of damage in cerebellar and 166 cortical structures, which have also been reported by studies using other methods 167 [19]. This observation raises the question of whether vitamin B5 depletion might be 168 cause or consequence of the underlying neuropathological process. We interpret 169 concomitant elevations in metabolites including glucose, glucose-6-phosphate, urea, 170 and the polyol pathway intermediates sorbitol and fructose, along with impaired 171 glucose uptake into the AD brain $[4,7,20]$, taken together with similar perturbations 172 in Huntington's disease brain [17, 21-23], to support the hypothesis that vitamin B5 
173 deficiency may be an early perturbation in the development of AD-evoked

174 neurodegeneration. The evident metabolic defects in the polyol and glycolytic

175 pathways could be linked to cerebral deficiency of vitamin B5, which could cause

176 defective formation of CoA and acetyl-CoA and uptake of acetyl-CoA into the TCA

177 cycle, along with downstream alterations in cerebral urea metabolism.

178 A ROC curve with AUC of above 0.9 (Fig. 2d) indicates high discriminatory power 179 of vitamin B5 concentration in classifying AD vs Control tissues. An optimal cutoff 180 value can be chosen based on the clinical requirements for sensitivity/specificity or 181 accuracy. In our data, the optimal cutoff value was 0.52 when given equal weight to 182 sensitivity and specificity (true positive rate $=0.86$, false positive rate $=0.19$ ). The 183 optimal cutoff value was 0.37 when accuracy was prioritized.

184 Vitamin B5 levels were not significantly correlated with age, PMD, or brain weight 185 (data not shown); Braak stage and amyloid load served as surrogates for the diagnosis 186 of AD (Suppl. Table 1), so their relationship to AD was not analysed further. 
Fig.1

a

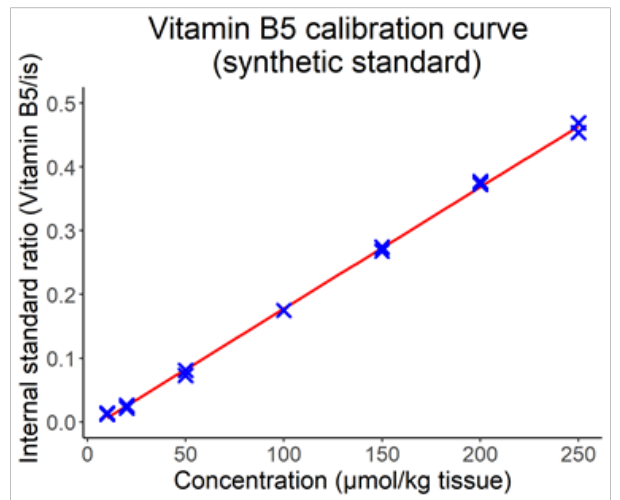

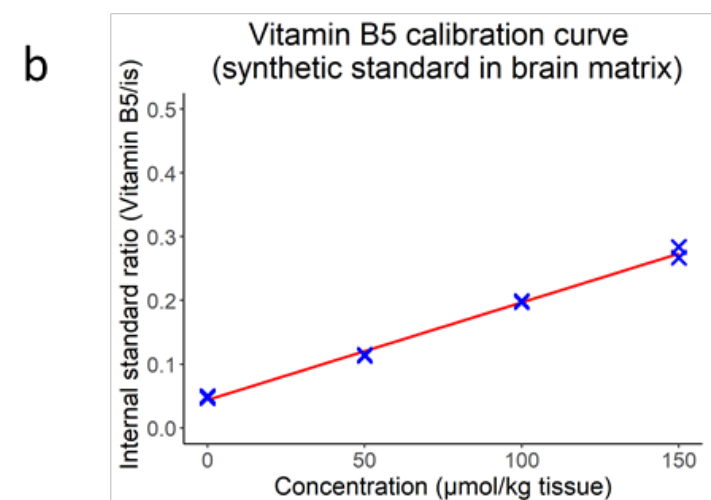

189 Figure 1. Calibration curve used for the determination of vitamin B5

190 concentrations in human brain. Calibration curves were generated by

191 measurements of known concentrations of synthetic Vitamin B5 standards in a)

192 aqueous medium, or b) in matrix generated from human-brain extract. 
Fig.2

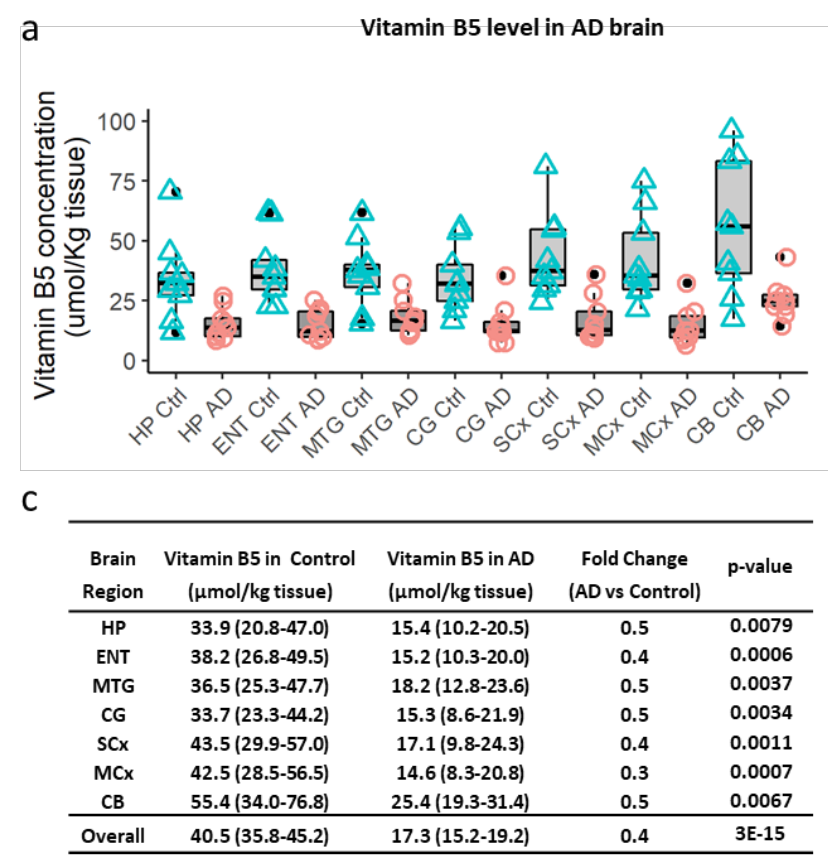

b

Vitamin B5 level in QC

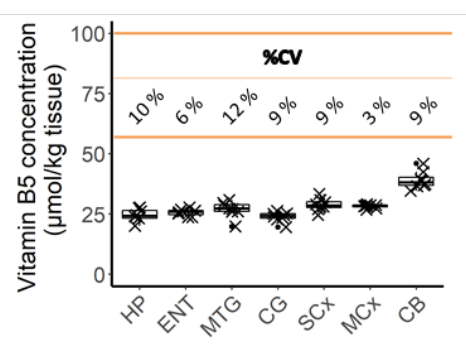

由 $A D$

官 Ctrl

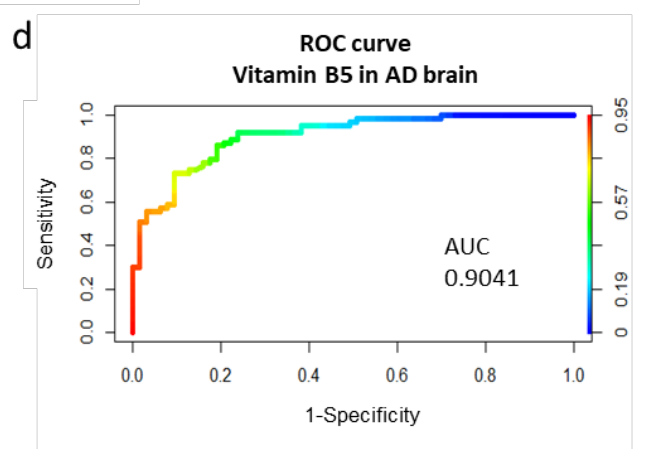

195

196 Figure 2. Vitamin B5 levels in human brain in cases with AD and matched

197 controls. a) Vitamin B5 concentrations measured in individual brain samples $(\mathrm{N}=$

198 18/region $=126$ in all); and b) in QC replicate samples; $c)$ mean $( \pm 95 \% \mathrm{CI})$ values of

199 vitamin B5 concentration for each brain region in controls and AD cases, where

200 statistical significance was calculated using multiple two-tailed $t$-tests; d) receiver-

201 operating characteristic curve for vitamin B5 among all brain samples. Abbreviations:

202 HP, hippocampus; ENT, entorhinal cortex; MTG, middle temporal gyrus; CG,

203 cingulate gyrus; SCx, sensory cortex; $\mathrm{MCx}$, motor cortex; $\mathrm{CB}$, cerebellum. 
205 Deficiency of several other B-group vitamins is known to associate with elevated risk 206 of dementia or other psychiatric disorders [24]: those B vitamins hitherto implicated 207 include thiamine (vitamin B1) [25]; niacin (vitamin B3) [26]; vitamin B6 (pyridoxal 208 phosphate; pyridoxamine; pyridoxine) [24]; folate (vitamin B9) [24]; and 209 cyanocobalamin (vitamin B12) [27, 28].

210 Here we hypothesize that severe, global deficiency of vitamin B5 in AD brain, as 211 observed in our current study, could contribute to defects in a number of molecular 212 pathways known to be implicated in the pathogenesis of neurodegeneration in $\mathrm{AD}$, in 213 particular those involving the metabolism of CoA and all those downstream pathways 214 that use CoA or its reduced form CoASH as an essential cofactor or biosynthetic 215 intermediate [29]: the latter roles include the cerebral biosynthesis of acetylcholine 216 and of myelin, both of which are deficient in $\mathrm{AD}[11,30-33]$. Therefore, cerebral 217 vitamin B5 deficiency is likely to be linked to the pathogenesis of dementia.

218 Notably, in our initial metabolomic study, vitamin B5 was the only B-vitamin whose 219 brain concentrations were measurable by the approach taken, and whose brain levels 220 were shown to be substantively deficient in AD dementia. More work will be required 221 to quantitate (putative) cerebral levels of the other B-vitamins in AD to further 222 characterise their possible relationship with the common age-related dementias.

223 The gene PANK2 encodes the enzyme PANK2 and regulates the utilization of vitamin 224 B5 through the pathway leading to the biosynthesis of CoA and of acetyl-CoA [34].

225 Mutations in PANK2 and associated PANK2 deficiency can cause PKAN through 226 impairing vitamin B5 metabolism and mitochondrial function, in turn leading to 227 mitochondrial CoA deficiency-associated oxidative stress. This chain of events 228 suggests that brain regions with higher metabolic demand could be particularly 229 susceptible to vitamin B5 deficiency.

230 Interestingly, brain regions corresponding to those heavily affected in AD also 231 showed relatively lower vitamin B5 levels in control brains. According to our 232 measurements in control brains, vitamin B5 levels ranged between 34 and $38 \mu \mathrm{mol} / \mathrm{kg}$ 233 of fresh tissue in those regions (hippocampus, entorhinal cortex, middle temporal 234 gyrus, and cingulate gyrus) that are heavily affected in AD; between 43 to $44 \mu \mathrm{mol} / \mathrm{kg}$ 235 in less affected brain regions (motor cortex and sensory cortex); and finally, 55 
$\mu \mathrm{mol} / \mathrm{kg}$ in the relatively-spared cerebellar region. Further study will be required to understand the mechanism and implications of these observations.

238 Vitamin B5 is the obligate precursor of acetyl-CoA, which is the substrate of the TCA

239 cycle generated by action of PDH. Reduced PDH complex and TCA-cycle activities

240 have previously been reported in $\mathrm{AD}$ brain $[35,36]$ and could be directly associated

241 with severe depletion of this acetyl-CoA precursor.

242 Acetyl-CoA plays numerous essential roles in cerebral metabolism. It is not only 243 required for energy production in mitochondria and diverse synthetic pathways in 244 various extra-mitochondrial compartments, but is also of particular importance for 245 cholinergic neurons in the brain: cholinergic neurons require additional amounts of 246 acetyl-CoA for acetylcholine synthesis in their cytoplasmic compartment to maintain 247 their transmitter functions [33]. Interestingly, the lowered PDH activity in AD brain 248 coincided with low choline acetyltransferase (CAT) activity [35]. Therefore it has 249 been suggested that deficits in acetyl-CoA supply may be more harmful for the 250 cholinergic neurons than for non-cholinergic ones in neurodegenerative diseases such 251 as AD [33]. Acetyl-CoA also plays key roles in biochemical pathways that underpin 252 the metabolism of fatty acids, porphyrins, polyamines, amino acids, proteins, RNA, 253 and histones; it is possible that alterations in any of the steps of the CoA biosynthetic 254 pathway might influence the proper functioning of any or all of such dependent 255 processes.

256 B-group vitamins are generally thought not to be stored in the brain; rather, specific 257 transporters are said to maintain the proper brain levels of each of these vitamins as 258 required by transferring them from the EC fluid. That said, vitamin B5 would seem to 259 be an exception, since large concentrations are present throughout the brain, where it 260 is localized largely with myelin in the white matter [11]. There is evidence that the 261 transporter SLC5A6, which is expressed in human-brain microvessels, is a major 262 contributor to luminal uptake of vitamin B5 and biotin at the human blood-brain 263 barrier and provides the main route for their uptake into brain [37]. In this study, brain 264 vitamin B5 levels in AD were lowered to 30-50\% of control values, so substantive 265 residual cerebral uptake via SLC5A6 must remain in AD-affected tissues; by contrast, 266 our hypothesis-generating metabolomic method did not produce a biotin-specific 267 signal. Vitamin B5 is ubiquitous in the diet, so dietary deficiency is not a plausible 
268 explanation for the observed cerebral deficiency in AD [10]. Vitamin B5 is well

269 tolerated when administered to humans in tablet form. Therefore, given the retention

270 of residual cerebral uptake, we expect it could be possible to restore brain vitamin B5

271 levels to normal by chronic administration, potentially reversing this abnormality and,

272 perhaps, the pathogenesis of AD.

273

274 In conclusion, there are evidently copious amounts of vitamin B5 throughout the

275 normal human brain, and AD dementia is accompanied by widespread deficiency

276 thereof. Vitamin B5 deficiency is perhaps more severe in those brain regions known

277 to be more severely damaged in AD. Vitamin B5 dysregulation could lie at the centre

278 of disturbed brain energetics, including the previously reported polyol-pathway

279 activation and concomitant impairment of glycolysis in AD brain [4]. We conclude

280 that cerebral vitamin B5 deficiency could cause neurodegeneration and dementia in

281 AD, which might be preventable, or even reversible in its early stages, by treatment

282 with oral doses of vitamin B5 sufficient to normalize brain levels.

\section{Duality of interest statement}

284 The authors state that they have no competing interests with respect to this work.

285 Sponsors had no role in the study design; the collection, analysis, and interpretation of 286 data; the writing of the manuscript, or the decision to submit the article for

287 publication. This work was generated during previous employment of JX and SP, and 288 not during their current employment.

289 Acknowledgements

290 We thank Dr Michael Anderson, The University of Manchester and Cynthia Tse, the 291 University of Auckland for their management of the research, and for proof-reading

292 and submission of the manuscript. We acknowledge the following funding sources:

293 the Endocore Research Trust (60187); the Oakley Mental Health Research

294 Foundation (NZ) $(3456030 ; 3627092 ; 3701339 ; 3703253 ; 3702879)$; the Maurice and

295 Phyllis Paykel Trust (3627036; and Travel funding for JX); the University of

296 Auckland (PReSS jxu58); the Maurice Wilkins Centre for Molecular Biodiscovery

297 (Tertiary Education Commission 9341-3622506); the University of Auckland

298 (Doctoral Scholarship for JX); the NZ Ministry of Business, Innovation \&

299 Employment (UoAX0815 Doctoral Scholarship for JX): the Medical Research 
300 Council (UK) Research Grant (MR/L010445/1); The University of Manchester; the 301 Northwest Regional Development Agency through a combined programme grant to 302 CADET; and the Greater Manchester Comprehensive Local Research Network. We 303 thank all the families of patients with Alzheimer's disease and controls, who so 304 generously supported this research through the donation of brain tissue to the 305 Neurological Foundation Human Brain Bank. 
[1] C.P. Ferri, M. Prince, C. Brayne, H. Brodaty, L. Fratiglioni, M. Ganguli, K. Hall, K. Hasegawa, H. Hendrie, Y. Huang, A. Jorm, C. Mathers, P.R. Menezes, E. Rimmer, M. Scazufca, Global prevalence of dementia: a Delphi consensus study, Lancet, 366 (2005) 2112-2117.

[2] M. Citron, Alzheimer's disease: strategies for disease modification, Nat Rev Drug Discov, 9 (2010) 387-398.

[3] S.M. de la Monte, M. Tong, Brain metabolic dysfunction at the core of Alzheimer's disease, Biochem Pharmacol, 88 (2014) 548-559. [4] J. Xu, P. Begley, S.J. Church, S. Patassini, S. McHarg, N. Kureishy, K.A. Hollywood, H.J. Waldvogel, H. Liu, S. Zhang, W. Lin, K. Herholz, C. Turner, B.J. Synek, M.A. Curtis, J. Rivers-Auty, C.B. Lawrence, K.A.B. Kellett, N.M. Hooper, E.R.L.C. Vardy, D. Wu, R.D. Unwin, R.L.M. Faull, A.W. Dowsey, G.J.S. Cooper, Elevation of brain glucose and polyol-pathway intermediates with accompanying brain-copper deficiency in patients with Alzheimer's disease: metabolic basis for dementia, Sci Rep, 6 (2016) 27524.

[5] Y. An, V.R. Varma, S. Varma, R. Casanova, E. Dammer, O. Pletnikova, C.W. Chia, J.M. Egan, L. Ferrucci, J. Troncoso, A. Levey, J. Lah, N.T. Seyfried, C. LegidoQuigley, O'Brien R., M. Thambisetty, Evidence for brain glucose dysregulation in Alzheimer's disease, Alzheimers Dement, 14 (2018) 318-329.

[6] R. Mullins, D. Reiter, D. Kapogiannis, Magnetic resonance spectroscopy reveals abnormalities of glucose metabolism in the Alzheimer's brain, Ann Clin Transl Neurol 5(2018) 262-272.

[7] J. Xu, P. Begley, S.J. Church, S. Patassini, K.A. Hollywood, M. Jüllig, M.A. Curtis, H.J. Waldvogel, R.L.M. Faull, R.D. Unwin, G.J.S. Cooper, Graded perturbations of metabolism in multiple regions of human brain in Alzheimer's disease: Snapshot of a pervasive metabolic disorder, Biochim Biophys Acta, 1862 (2016) 10841092.

[8] J. Xu, S. Patassini, N. Rustogi, I. Riba-Garcia, B.D. Hale, A.M. Phillips, H. Waldvogel, R. Haines, P. Bradbury, A. Stevens, R.L.M. Faull, A.W. Dowsey, G.J.S. Cooper, R.D. Unwin, Regional protein expression in human Alzheimer's brain correlates with disease severity, Commun Biol, 2 (2019) 43.

[9] C. Choudhary, B.T. Weinert, Y. Nishida, E. Verdin, M. Mann, The growing landscape of lysine acetylation links metabolism and cell signalling, Nat Rev Mol Cell Biol, 15 (2014) 53.

[10] R.B. Rucker, K. Bauerly, Pantothenic acid, in: J. Zempleni, J.W. Suttie, J.F. Gregory III, P.J. Stover (Eds.) Handbook of Vitamins, Fifth Edition, CRC Press, Boca Raton, FL, USA, 2014, pp. 325-349.

[11] N. Ismail, N. Kureishy, S.J. Church, M. Scholefield, R.D. Unwin, J. Xu, S. Patassini, G.J.S. Cooper, Vitamin B5 (D-pantothenic acid) localizes in myelinated structures of the rat brain: potential role for cerebral vitamin B5 stores in local myelin homeostasis, Biochem Biophys Res Commun, 552 (2020) 220-225. [12] M. Daugherty, B. Polanuyer, M. Farrell, M. Scholle, A. Lykidis, V. de CrecyLagard, A. Osterman, Complete reconstitution of the human coenzyme A biosynthetic pathway via comparative genomics, J Biol Chem, 277 (2002) 21431-21439. 

S.J. Hayflick, Mitochondrial localization of human PANK2 and hypotheses of secondary iron accumulation in pantothenate kinase-associated neurodegeneration, Ann N Y Acad Sci, 1012 (2004) 282-298. [14] K.A. Jobst, A.D. Smith, M. Szatmari, M.M. Esiri, A. Jaskowski, N. Hindley, B. McDonald, A.J. Molyneux, Rapidly progressing atrophy of medial temporal lobe in Alzheimer's disease, Lancet, 343 (1994) 829-830.

360 [15] K.M. Bradley, V.T. O'Sullivan, N.D. Soper, Z. Nagy, E.M. King, A.D. Smith, B.J. Shepstone, Cerebral perfusion SPET correlated with Braak pathological stage in Alzheimer's disease, Brain, 125 (2002) 1772-1781. [16] H. Braak, E. Braak, Neuropathological stageing of Alzheimer-related changes, Acta Neuropathol, 82 (1991) 239-259.

[17] S. Patassini, P. Begley, J. Xu, S.J. Church, S.J. Reid, H.J. Waldvogel, R.G. Snell, R.L.M. Faull, R.D. Unwin, G.J.S. Cooper, Cerebral vitamin B5 (D-pantothenic acid) deficiency as a potential cause of metabolic perturbation and neurodegeneration in Huntington's disease, Metabolites, 9 (2019) 113.

[18] I. Skoog, Detection of preclinical Alzheimer's disease, N Engl J Med, 343 (2000) 502-503.

371 [19] C.C. Guo, R. Tan, J.R. Hodges, X. Hu, S. Saber, M. Hornberger, Networkselective vulnerability of the human cerebellum to Alzheimer's disease and frontotemporal dementia, Brain, 139 (2016) 1532-1543.

[20] L. Mosconi, W.H. Tsui, K. Herholz, A. Pupi, A. Drzezga, G. Lucignani, E.M. Reiman, V. Holthoff, E. Kalbe, S. Sorbi, J. Diehl-Schmid, R. Perneczky, F. Clerici, R. Caselli, B. Beuthien-Baumann, A. Kurz, S. Minoshima, M.J. de Leon, Multicenter standardized ${ }^{18}$ F-FDG PET diagnosis of Mild Cognitive Impairment, Alzheimer's disease, and other dementias, J Nucl Med, 49 (2008) 390-398.

[21] S. Patassini, P. Begley, S.J. Reid, J. Xu, S.J. Church, M. Curtis, M. Dragunow, H.J. Waldvogel, R.D. Unwin, R.G. Snell, R.L.M. Faull, G.J.S. Cooper, Identification of elevated urea as a severe, ubiquitous metabolic defect in the brain of patients with Huntington's disease, Biochem Biophys Res Commun, 468 (2015) 161-166. [22] S. Patassini, P. Begley, J. Xu, S.J. Church, S.J. Reid, E.H. Kim, M.A. Curtis, M. Dragunow, H.J. Waldvogel, R.G. Snell, R.D. Unwin, R.L.M. Faull, G.J.S. Cooper, Metabolite mapping reveals severe widespread perturbation of multiple metabolic processes in Huntington's disease human brain, Biochim Biophys Acta, 1862 (2016) 1650-1662.

[23] R.R. Handley, S.J. Reid, R. Brauning, P. Maclean, E.R. Mears, I. Fourie, S. Patassini, G.J.S. Cooper, S.R. Rudiger, C.J. McLaughlan, P. Verma, J.F. Gusella, M.E. MacDonald, H.J. Waldvogel, C.S. Bawden, R.L.M. Faull, R.G. Snell, Brain urea increase is an early Huntington's disease pathogenic event observed in a prodromal transgenic sheep model and HD cases, Proc Natl Acad Sci USA, 114 (2017) E11293-E11302.

[24] M.C. Morris, J.A. Schneiderd, C.C. Tangney, Thoughts on B-vitamins and dementia, J Alzheimers Dis, 9 (2006) 429-433.

[25] G.E. Gibson, J.A. Hirsch, P. Fonzetti, B.D. Jordan, R.T. Cirio, J. Elder, Vitamin B1 (thiamine) and dementia, Ann N Y Acad Sci, 1367 (2016) 21-30.

[26] M.C. Morris, D.A. Evans, J.L. Bienias, P.A. Scherr, C.C. Tangney, L.E. Hebert, D.A. Bennett, R.S. Wilson, N. Aggarwal, Dietary niacin and the risk of incident 
400 Alzheimer's disease and of cognitive decline, J Neurol Neurosurg Psychiatry, 75

401 (2004) 1093-1099.

402 [27] R. Malouf, J. Grimley Evans, Folic acid with or without vitamin B12 for the 403 prevention and treatment of healthy elderly and demented people (Review),

404 Cochrane Database Syst Rev, (2008) Art. No.: CD004514.

405 [28] E.H. Reynolds, Folic acid, ageing, depression, and dementia, BMJ (Clinical 406 research ed.), 324 (2002) 1512-1515.

407 [29] D.O. Kennedy, B vitamins and the brain: mechanisms, dose and efficacy-a 408 review, Nutrients, 8 (2016) 68.

409 [30] E. Fornaria, P. Maedera, R. Meulia, J. Ghikac, M.G. Knyazevaa, Demyelination 410 of superficial white matter in early Alzheimer's disease: a magnetization transfer 411 imaging study, Neurobiol Aging, 33 (2012) 428.e427- 428.e419.

412 [31] S. Mitew, M.T.K. Kirkcaldie, G.M. Halliday, C.E. Shepherd, J.C. Vickers, T.C. 413 Dickson, Focal demyelination in Alzheimer's disease and transgenic mouse 414 models, Acta Neuropathol, 119 (2010) 567-577.

415 [32] R.D. Fields, D.J. Dutta, J. Belgrad, M. Robnett, Cholinergic signaling in 416 myelination, Glia, 65 (2017) 687-698.

417 [33] A. Szutowicz, H. Bielarczyk, A. Jankowska-Kulawy, T. Pawelczyk, A.

418 Ronowska, Acetyl-CoA the key factor for survival or death of cholinergic neurons 419 in course of neurodegenerative diseases, Neurochem Res, 38 (2013) 1523-1542.

420 [34] S.J. Hayflick, Defective pantothenate metabolism and neurodegeneration, 421 Biochem Soc Trans, 42 (2014) 1063-1068.

422 [35] S. Sorbi, E.D. Bird, J.P. Blass, Decreased pyruvate dehydrogenase complex 423 activity in Huntington and Alzheimer brain, Ann Neurol, 13 (1983) 72-78.

424 [36] P. Bubber, V. Haroutunian, G. Fisch, J.P. Blass, G.E. Gibson, Mitochondrial 425 abnormalities in Alzheimer brain: mechanistic implications, Ann Neurol, 57 426 (2005) 695-703.

427 [37] Y. Uchida, K. Ito, S. Ohtsuki, Y. Kubo, T. Suzuki, T. Terasaki, Major 428 involvement of $\mathrm{Na}(+)$-dependent multivitamin transporter (SLC5A6/SMVT) in 429 uptake of biotin and pantothenic acid by human brain capillary endothelial cells, 430 J Neurochem, 134 (2015) 97-112. 